\title{
Ecological Momentary Assessment based Differences between Android and iOS Users of the TrackYourHearing mHealth Crowdsensing Platform
}

\author{
Rüdiger Pryss ${ }^{1}$, Winfried Schlee ${ }^{2}$, Manfred Reichert ${ }^{1}$, Ira Kurthen ${ }^{3}$, Nathalie Giroud ${ }^{4}$, \\ Laura Jagoda $^{5}$, Pia Neuschwander ${ }^{5}$, Martin Meyer ${ }^{5}$, Patrick Neff ${ }^{2}$, Johannes Schobel ${ }^{1}$, \\ Burkhard Hoppenstedt ${ }^{1}$, Myra Spiliopoulou ${ }^{6}$, Berthold Langguth ${ }^{2}$, and Thomas Probst ${ }^{7}$
}

\begin{abstract}
Health technologies are increasingly utilized in various medical contexts. Mobile crowdsensing is such a technology, which is often used for data collection scenarios related to questions on chronic disorders. One prominent reason for the latter setting is based on the fact that powerful Ecological Momentary Assessments (EMA) can be performed. Notably, when mobile crowdsensing solutions are used to integrate EMA measurements, many new challenges arise. For example, the measurements must be provided in the same way on different mobile operating systems. However, the newly given possibilities can surpass the challenges. For example, if different mobile operating systems must be technically provided, one direction could be to investigate whether users of different mobile operating systems pose a different behaviour when performing EMA measurements. In a previous work, we investigated differences between iOS and Android users from the TrackYourTinnitus mHealth crowdsensing platform, which has the goal to reveal insights on the daily fluctuations of tinnitus patients. In this work, we investigated differences between iOS and Android users from the TrackYourHearing mHealth crowdsensing platform, which aims at insights on the daily fluctuations of patients with hearing loss. We analyzed 3767 EMA measurements based on a daily applied questionnaire of 84 patients. Statistical analyses have been conducted to see whether these 84 patients differ with respect to the used mobile operating system and their given answers to the EMA measurements. We present the obtained results and compare them to the previous mentioned study. Our insights show the differences in the two studies and that the overall results are worth being investigated in a more indepth manner. Particularly, it must be investigated whether the used mobile operating system constitutes a confounder when gathering EMA-based data through a crowdsensing platform.

Index Terms - mHealth, crowdsensing, hearing loss, EMA
\end{abstract}

\footnotetext{
${ }^{1}$ Faculty of Computer Science, Engineering and Psychology, Ulm University, Germany

\{ruediger.pryss, manfred.reichert, burkhard.hoppenstedt, johannes.schobel

${ }^{2}$ University Hospital Regensburg, Germany winfried.schlee@ieee.org, berthold.langguth@medbo.de, patrick.neff@ukr. de

${ }^{3}$ Developmental Psychology: Infancy and Childhood, Department of Psychology, University of Zurich, Zurich, Switzerland i.kurthenepsychologie.uzh.ch

${ }^{4}$ Cognition, Aging, and Psychophysiology Laboratory, Department of Psychology, Concordia University, Montreal, Canada nathalie.giroudehotmail.com

${ }^{5}$ Division of Neuropsychology, Department of Psychology, University of Zurich, Zurich, Switzerland pia.neuschwander@uzh.ch, laura.jagoda@psychologie.uzh.ch, m.meyer@psychologie.uzh.ch

${ }^{6}$ University of Magdeburg, Germany myra@ovgu. de

${ }^{7}$ Department for Psychotherapy and Biopsychosocial Health, Danube University Krems, Austria Thomas.Probstedonau-uni.ac.at
}

\section{INTRODUCTION}

Mobile crowdsensing is a technology that has proven its worth for the medical domain. For example, in previous works, we found interesting insights on data gathered with the TrackYourTinnitus (TYT) mHealth crowdsensing platform [1], [2]. Also other works have revealed new insights when using mobile crowdsensing in the context of chronic diseases [3]. In general, these findings are based on measurements in daily life, i.e., ambulatory assessments / Ecological Momentary Assessments (EMA). These measurements can be realized by built-in sensors of modern smartphones and the utilization of electronic questionnaires, which are filled out by the users on the smartphone repeatedly over a longer period of time [4]. Following this, new medical datasets become possible [5]. However, the technical realization of a platform that properly enables the aforementioned aspects is a challenging endeavor. Based on experiences when running TYT [6]-[8] for years, we were able to adjust the technical platform to other healthcare questions. So far, the technology was adjusted to medical questions on the loss of hearing, the management of stress and diabetes as well as the support of pregnant women (see Table I). For data that was gathered with TYT, we revealed investigation opportunities that were not thought of when designing the technology, e.g., to compare retrospective and prospective statements of tinnitus patients [7]. Another observation that was not initially intended constitutes the opportunity to compare the behavior of TYT users with respect to their used mobile operating system. Whether a user has given an answer with an Android or iOS smartphone was by design only recorded for testing purposes.

However, it emerged that this information can also be used to compare differences between Android and iOS users with respect to their demographic and health characteristics

\begin{tabular}{lll}
\hline Project & Medical Aspect & URL \\
\hline TrackYourTinnitus & Tinnitus & http://www.trackyourtinnitus.org \\
TrackYourHearing & Hearing Loss & http://www.trackyourhearing.org \\
TrackYourStress & Stress & http://www.trackyourstress.org \\
Chrodis+ & Diabetes & http://chrodis.eu \\
MyKind & Pregnant Women & http://www.mykind.info \\
\hline
\end{tabular}

TABLE I: mHealth Crowdsensing Projects 
on tinnitus [9]. In this work, in turn, we took a closer look at the current dataset of the TrackYourHearing (TYH) mHealth crowdsensing platform, which addresses the daily fluctuations of users with a hearing loss. Note that the latter constitutes one of the top causes of years lived with a disability [10]. The investigation we conducted in this work aims at two goals:

- Can we reveal differences between Android and iOS users of the TrackYourHearing mHealth crowdsensing platform based on their collected EMA data?

- Can we confirm the results of the TrackYourTinnitus mHealth crowdsensing platform on differences between Android and iOS users as shown in [9]?

The remainder of this paper is organized as follows. In Section [II, relevant related work will be reviewed. Section III] in turn, provides background information on the TrackYourHearing mHealth crowdsensing platform. In Section V, the materials and methods used for the data analysis are described. Section VI presents the obtained results, while Section VI discusses them. Section VIII finally concludes the paper with a summary and an outlook.

\section{RELATED WORK}

Three categories of related work are relevant in the context of this work: (1) Approaches that deal with mobile crowdsensing and EMA in the healthcare domain, (2) approaches on differences between Android and iOS in the context of mobile healthcare applications, and (3) mobile applications focusing on hearing loss. Regarding the first category, some recent works exist that deal with generic crowdsensing approaches to enable human-subject studies [11]. However, the use of crowdsensing in the context of chronic diseases is still rare. One example for chronic disorders is the TrackYourTinnitus mHealth crowdsensing platform [1], [2], [12]. In turn, technical solutions that enable EMA measurements without using mobile crowdsensing technology have been presented with valuable healthcare results [13], [14].

Regarding the second category, approaches that compare differences between Android and iOS in the context of mobile healthcare applications are of particular interest. In [15], [16], for example, security issues for Android and iOS mHealth applications are discussed. The authors of [17], in turn, discuss guidelines of Android and iOS to create applications that are able to support personal health records. In general, many works exist that aim to evaluate the differences and quality of mHealth applications that were developed for both mobile operating systems [18]-[20]. Approaches that directly compare Android and iOS users exist beyond healthcare questions [21]. Android and iOS users were directly compared only in a few studies [22]. These studies have found that iOS users are more likely female, in the mid-30s, with a graduate degree, in a higher income group, with more technology knowledge, and that iOS users spend more time using applications than Android users. Clinically relevant differences were reported for the SmokeFree28 (SF28) application [22]. For example, iOS users downloaded the app more likely for a serious attempt to quit smoking. Android users, in turn, took stop-smoking medication more often. Another study investigated whether iOS and Android users differ in personality traits [23]. Only a few and small differences were found. Altogether, research on mobile crowdsensing based differences between Android and iOS users is still in its infancy.

Regarding the third category, approaches exist that deal with mobile technology in the context of hearing loss [24], [25]. However, these works do not directly measure parameters on the hearing loss, they rather measure parameters that may negatively affect the onset of a hearing loss (e.g., through a loud environment). In the context of EMA and mobile technology in general, other works exist that have shown its usefulness for healthcare questions on hearing loss [26]. Finally, systematic literature reviews show that many mobile apps beside mobile crowdsensing exist that address a hearing loss [27], [28]. However, to the best of our knowledge, none of these works have compared Android and iOS users as shown in the work at hand.

\section{Track YourHeAring Platform}

Mobile crowdsensing is characterized by the following aspects. Different sensing paradigms are utilized to relate crowd users to sensing tasks on one hand [29]. On the other, a sophisticated crowdsensing platform must be developed to enable measurements by crowd users. In the context of mHealth questions, three technical components are particularly necessary to provide a proper crowdsensing platform. First, a proper data model including a flexible API to handle the data exchange must be developed [8]. Second, an architecture must be defined that reflects the needs for the collection procedure [11]. Finally, mobile apps must be realized that enable a collection procedure that is welcome by the users [30]. These aspects are considered by the TrackYourHearing (TYH; https://www.trackyourhearing.org) mHealth crowdsensing platform, which is built on four technical components. First, it offers a website for user registration and other user-related features (e.g., account management). Second, it offers an Android and iOS application. Third, a MySQL database is used for the central repository for the data collected. Fourth, a RESTful API is provided that enables the communication between the mobile applications, the website, and the database [8].

In general, TYH was developed to collect EMA of individuals with a hearing loss. On the one hand, EMA is based on a set of electronic questionnaires, which are repeatedly (registration, daily) administered to the users on their smartphones. On the other hand, EMA is based on sensorbased measurements of the environmental sound level. Yet, the measurement of the environmental sound level is only realized if users actively give consent for this measurement when registering to TYH for the first time. In doing so, we consider the privacy of the users. In general, TYH users accomplish three fundamental phases. First, they have to register through the website or the mobile apps. Second, users have to fill in three so-called registration questionnaires once. The latter capture the current hearing loss situation, 
demographic data (e.g., birthday), and other hearing loss related parameters. The completion of these registration questionnaires is compelling for the users who want to use the features of the continuous mobile crowdsensing procedure (i.e., the daily measurements). Also, during the second phase, users have to accept or adjust a notification schema. The notification schema determines how often and in what way (i.e., fixed or random points in time) the daily assessment questionnaire is applied. The number of daily assessments, in turn, is restricted to 12 times per day. Third, after the registration questionnaires have been accomplished and the notification schema is determined, users can start with the daily assessments. For the application of the daily assessment questionnaire, notification features for both Android and iOS, as well as a notification algorithm, were realized. After a notification appears, the user may click on it. In the latter case, the mobile application is started (if not already running) and the daily assessment questionnaire is directly displayed to the user for completion. Note that users can also fill in the questionnaires without a notification whenever they want. While filling out this questionnaire, the environmental sound level is measured in users who agreed to this. The result is then either transferred through the API to the database or locally cached if the device is offline after completing the questionnaire. A more detailed technical description of the presented features can be found in [7], [8], [31].

\section{Material And Methods}

This section provides materials and methods that were used for analyses. Note that TYH is currently only available in German, while English and French versions are under development. The mobile apps have been officially released to the Google Playstore and the Apple App Store. So far, users that have been registered to TYH mainly stem from Switzerland 54\%, Germany $22 \%$, and the USA $6 \%$. For the presented analysis, results of the daily assessment questionnaires were mainly used, for which the questions are shown in Table II It is noteworthy that three types of user interface elements were used to give answers: sliders to enable visual analogue scales (VAS), yes/no questions as well as self-assessment manikins (SAM; [32]). In the first data preparation step, all test users were removed, resulting in 84 users with 3767 filled out daily assessment questionnaires, with an inter-assessment interval of at least 15 minutes. In addition, we analyzed one of the registration questionnaires, which comprises five questions on gender, handedness, age, and whether a patient wears a hearing aid, and whether a patient has actually a hearing loss. This questionnaire was filled out by all of the 84 analyzed users.

\section{STATISTICS}

All statistical analyses were performed with SPSS 25. Means (M) and standard deviations (SD) were calculated as descriptive statistics. Assessments with an inter-assessment interval $<15$ minutes were deleted. Android and iOS users were compared with Fishers Exact Tests (FET), t-tests for independent samples, and multilevel models. To analyze the

\begin{tabular}{clc} 
& Question & Scale \\
\hline (1) & Do you wear your hearing aid right now? & Y/N \\
(2) & To what extent do you perceive your hearing loss right now? & VAS \\
(3) & To what extent are you limited in your daily life by your hearing & VAS \\
(4) & loss right now? & \\
(5) & How is your mood right now? & VAS \\
(6) & Do you feel stressed right now? & VAS \\
(7) & Do you feel irritable right now? & VAS \\
(8) & Do you feel exhausted right now? & VAS \\
(9) & To what extent were conversations of the last hours burdensome? & VAS \\
(2) & Regarding the latter question, if you had no conversations, please & Y/N \\
(1) & Do you physically perceive ambient noises negatively right now? & VAS \\
\hline & VAS=Visual Analogue Scale, Y/N=Yes/No-Question \\
& SAM=Self-Assessment Manikins & \\
\hline
\end{tabular}

TABLE II: TrackYourHearing Daily Assessment Questions

items of the registration questionnaire, which was provided once to the users, FET and t-tests were used. T-tests were also used to analyze the following variables: days between first and last daily assessment questionnaire within a user, amount of daily assessment questionnaires per user, and hours between each of the daily assessment questionnaire across users. Linear multilevel models with two levels were used for the items of the daily assessment questionnaire with a numeric rating scale (i.e., Questions 2-10, except Question 9b). These repeated daily assessments are nested within users, so that assessments were level-1 and users level-2 in the multilevel models. The linear multilevel models were performed with the full maximum likelihood estimation and a random intercept. For the binary Questions 1 and $9 \mathrm{~b}$, which show again the mentioned nested data structure, multilevel models for dichotomous outcomes, i.e., binary logistic models within the Generalized Estimating Equations (GEE) were performed. In all multilevel models, the main effect of the mobile operating system (Android vs. iOS with Android being the reference coded as 0 and iOS coded as 1) was evaluated and the scores of the items of the daily assessment questionnaires functioned as the dependent variables. All statistical tests were performed two-tailed. The significance value was set to $p<.05$.

\section{RESUlTS}

In total, $N=84$ users participated in the analysis. All users filled in the registration questionnaire. In total, the users 3767 daily assessment questionnaires. Of the $N=84$ users, $n=37$ used iOS, while $n=47$ used Android. The Android users provided 2450 daily assessment questionnaires and the iOS users 1317. Table III presents the results of the comparison between Android and iOS users with t-tests and FET, which were performed to compare the users in the registration questionnaire items. No significant differences between Android and iOS emerged. Table IV] shows the results of the multilevel models comparing the Android and iOS users in their scores of the daily assessment questionnaires. In three questions, iOS users scored significantly higher (all $p<.05$ ) than Android users: Questions 7 (irritable), 8 (exhausted), and 10 (ambient noises negative). Scores on Question 1 


\begin{tabular}{|c|c|c|c|c|}
\hline & & Android & iOS & Statistics \\
\hline \multirow{2}{*}{ Gender } & male & $25(53.2 \%)$ & $18(48.6 \%)$ & \multirow{2}{*}{ FET: $\mathrm{p}=.826$} \\
\hline & female & $22(46.8 \%)$ & $19(51.4 \%)$ & \\
\hline \multirow{4}{*}{ Hearing Ability } & no problem & $16(34.0 \%)$ & $14(37.8 \%)$ & \multirow{4}{*}{ FET: $\mathrm{p}=.875$} \\
\hline & problem in both ears & $27(57.4 \%)$ & $21(56.8 \%)$ & \\
\hline & problem in right ear & $1(2.1 \%)$ & $1(2.7 \%)$ & \\
\hline & problem in left ear & $3(6.4 \%)$ & $1(2.7 \%)$ & \\
\hline \multirow{2}{*}{ Hearing Aid } & no & $37(78.7 \%)$ & $23(62.2 \%)$ & \multirow{2}{*}{ FET: $\mathrm{p}=.144$} \\
\hline & yes & $10(21.3 \%)$ & $14(37.8 \%)$ & \\
\hline \multirow{3}{*}{ Handedness } & ambidextrous & $0(0 \%)$ & $1(2.7 \%)$ & \multirow{3}{*}{ FET: $\mathrm{p}=.207$} \\
\hline & left & $1(2.1 \%)$ & $3(8.1 \%)$ & \\
\hline & right & $46(97.9 \%)$ & $33(89.2 \%)$ & \\
\hline \multicolumn{2}{|c|}{$\begin{array}{l}\text { Days between first and last daily } \\
\text { assessment questionnaire within a user M (SD) }\end{array}$} & $26.26(38.68)$ & $20.09(20.23)$ & $\mathrm{T}(82)=.88 ; \mathrm{p}=.382$ \\
\hline \multicolumn{2}{|l|}{ Age M (SD) } & $67.84(12.55)$ & $67.06(9.32)$ & $\mathrm{T}(81)=.31 ; \mathrm{p}=.755$ \\
\hline \multicolumn{2}{|c|}{ Amount of daily assessment questionnaires per user M (SD) } & $52.13(67.64)$ & $35.59(31.00)$ & $\mathrm{T}(82)=1.38 ; \mathrm{p}=.173$ \\
\hline \multicolumn{2}{|c|}{$\begin{array}{l}\text { Hours between each of the daily assessment questionnaires } \\
\text { across users M (SD) }\end{array}$} & $11.83(78.68)$ & $13.46(53.00)$ & $\mathrm{T}(3681)=-.67 ; \mathrm{p}=.506$ \\
\hline
\end{tabular}

TABLE III: Results of the Fishers Exact Tests and t-tests

\begin{tabular}{|l|l|l|l|}
\hline & & $\begin{array}{l}\text { Android } \\
(\text { Intercept })\end{array}$ & $\begin{array}{l}\text { iOS } \\
\text { (Alteration compared to Android) }\end{array}$ \\
\hline \multirow{2}{*}{ Question 2} & Estimate (SE) & $.11(.02)$ & $.04(.02)$ \\
\cline { 2 - 4 } & Statistics & $\mathrm{T}(77.56)=6.67 ; \mathrm{p}<.001$ & $\mathrm{~T}(79.10)=1.66 ; \mathrm{p}=.101$ \\
\hline \multirow{2}{*}{ Question 3} & Estimate (SE) & $.11(.02)$ & $.04(.03)$ \\
\cline { 2 - 4 } & Statistics & $\mathrm{T}(76.96)=6.42 ; \mathrm{p}<.001$ & $\mathrm{~T}(78.09)=1.57 ; \mathrm{p}=.120$ \\
\hline \multirow{2}{*}{ Question 4} & Estimate (SE) & $.10(.02)$ & $.04(.03)$ \\
\cline { 2 - 4 } & Statistics & $\mathrm{T}(75.50)=5.87 ; \mathrm{p}<.001$ & $\mathrm{~T}(77.40)=1.60 ; \mathrm{p}=.114$ \\
\hline \multirow{2}{*}{ Question 5 } & Estimate (SE) & $.79(.02)$ & $-.05(.03)$ \\
\cline { 2 - 4 } & Statistics & $\mathrm{T}(75.13)=39.26 ; \mathrm{p}<.001$ & $\mathrm{~T}(77.33)=-1.67 ; \mathrm{p}=.100$ \\
\hline \multirow{2}{*}{ Question 6 } & Estimate (SE) & $.11(.02)$ & $.03(.02)$ \\
\cline { 2 - 4 } & Statistics & $\mathrm{T}(77.90)=7.45 ; \mathrm{p}<.001$ & $\mathrm{~T}(80.13)=1.11 ; \mathrm{p}=.269$ \\
\hline \multirow{2}{*}{ Question 7 } & Estimate (SE) & $.13(.02)$ & $.61(.03)$ \\
\cline { 2 - 4 } & Statistics & $\mathrm{T}(76.33)=5.52 ; \mathrm{p}<.001$ & $\mathrm{~T}(79.23)=17.72 ; \mathrm{p}<.001$ \\
\hline \multirow{2}{*}{ Question 8} & Estimate (SE) & $.15(.02)$ & $.08(.03)$ \\
\cline { 2 - 4 } & Statistics & $\mathrm{T}(70.18)=6.96 ; \mathrm{p}<.001$ & $\mathrm{~T}(72.08)=2.42 ; \mathrm{p}=.018$ \\
\hline \multirow{2}{*}{ Question 9a } & Estimate (SE) & $.15(.02)$ & $.04(.03)$ \\
\cline { 2 - 4 } & Statistics & $\mathrm{T}(78.76)=7.43 ; \mathrm{p}<.001$ & $\mathrm{~T}(80.41)=1.20 ; \mathrm{p}=.235$ \\
\hline \multirow{2}{*}{ Question 10 10} & Estimate (SE) & $.11(.02)$ & $.07(.03)$ \\
\cline { 2 - 4 } & Statistics & $\mathrm{T}(77.27)=6.59 ; \mathrm{p}<.001$ & $\mathrm{~T}(79.75)=2.61 ; \mathrm{p}=.011$ \\
\hline
\end{tabular}

TABLE IV: Results of the Linear Multilevel Models

were not significantly different for Android and iOS users $(p=.489)$, as were scores for Question 9b $(p=.779)$.

\section{DISCUSSION}

Compared to our analysis on TYT and differences between Android and iOS users [9], this work has revealed different results. First of all, the registration questionnaire that was used for TYH showed no difference for Android and iOS users. For the variable age, significant differences were obtained for TYT, but not for TYH. As we have much less daily assessments gathered by TYH than TYT users, we firstly had a look at the daily assessment questionnaire of TYH and whether there exist differences before analyzing the other two registration questionnaires. Table IV shows that there are significant differences for three questions of the daily questionnaire between Android and iOS users. Therefore, it is worth to investigate the other registration questionnaires of TYH. However, it must be considered that the amount of assessments for TYH is sparse compared to TYT and a larger sample must be reconsidered for TYH. For TYT, in turn, differences between Android and iOS users on the daily assessment questionnaire have not been 
investigated so far [9]. Another observation that was new compared to the study on TYT is to have a look at the amount of assessments users provided per mobile operating system on average. As can be obtained from Table 1, Android users provide apparently more daily assessments (i.e., daily assessment) than $\mathrm{iOS}$ users do, although there is no significant difference ascertainable. Referring to the two questions raised in the introduction, we can conclude as follows: Concerning Question 1, we found significant differences of Android and iOS users for TYH based on their given EMA-data. Concerning Question 2, this study found differences between Android and iOS users, but not the same differences as the previous study on TYT [9].

\section{SUMMARY AND OUTLOOK}

Although this work on TYH has shown different results to the previous study on TYT, an investigation with the goal to reveal medically relevant differences between Android and iOS users based on EMA-data gathered by a crowdsensing platform should be generally taken into account. In particular, it must be further worked on the question whether the used mobile operating system can reveal new insights and whether it should be considered as a potential confounder in future studies. However, although TYH has a smaller database compared to TYT, again differences between Android and iOS users have been obtained. Therefore, we currently focus our research on various different directions. One direction constitutes the application of machine learning techniques to see whether we are able to predict the used mobile operating system based on daily EMA-data. Another direction is an indepth investigation on how the user interfaces of the different mobile operating systems may bias the user when filling out EMA questionnaires. Altogether, differences of Android and iOS users in the context of mHealth and chronic disorders can be considered as a promising target in general.

\section{REFERENCES}

[1] W. Schlee et al., "Measuring the moment-to-moment variability of tinnitus: the trackyourtinnitus smart phone app," Frontiers in Aging Neuroscience, vol. 8, 2016.

[2] T. Probst et al., "Emotional States as Mediators between Tinnitus Loudness and Tinnitus Distress in Daily Life: Results from the "TrackYourTinnitus" application," Scientific Reports, vol. 6, 2016.

[3] A. Vasilateanu, I. Radu, and A. Buga, "Environment crowd-sensing for asthma management," in E-Health and Bioengineering Conference. IEEE, 2015, pp. 1-4.

[4] T. Trull and U. Ebner-Priemer, "Ambulatory assessment," Annual review of clinical psychology, vol. 9, pp. 151-176, 2013.

[5] S. Engel et al., "Ecological momentary assessment in eating disorder and obesity research: a review of the recent literature," Current psychiatry reports, vol. 18, no. 4, pp. 1-9, 2016.

[6] R. Pryss et al., "Mobile Crowd Sensing Services for Tinnitus Assessment, Therapy and Research," in IEEE 4th Int'l Conf on Mobile Services. IEEE Computer Society Press, 2015.

[7] —, "Prospective crowdsensing versus retrospective ratings of tinnitus variability and tinnitus-stress associations based on the trackyourtinnitus mobile platform," International Journal of Data Science and Analytics, pp. 1-12, 2018.

[8] —, "Requirements for a flexible and generic api enabling mobile crowdsensing mhealth applications," in 4th Int'l WS on Requirements Engineering for Self-Adaptive, Collaborative, and Cyber Physical Systems. IEEE, 2018, pp. 24-31.
[9] —-, "Differences between Android and iOS Users of the TrackYourTinnitus Mobile Crowdsensing mHealth Platform," in Proc. 31th IEEE International Symposium on Computer-Based Medical Systems. IEEE Computer Society Press, 2018.

[10] T. Vos et al., "Global, regional, and national incidence, prevalence, and years lived with disability for 328 diseases and injuries for 195 countries, 1990-2016: a systematic analysis for the global burden of disease study 2016," The Lancet, vol. 390, no. 10100, pp. 1211-1259, 2017.

[11] H. Xiong et al., "Sensus: a cross-platform, general-purpose system for mobile crowdsensing in human-subject studies," in Proc of the Int'l Conf on Pervasive and Ubiquitous Computing. ACM, 2016, pp. 415-426.

[12] T. Probst et al., "Emotion dynamics and tinnitus: Daily life data from the "trackyourtinnitus" application," Scientific reports, vol. 6, 2016.

[13] U. Ebner-Priemer and T. Kubiak, "Psychological and psychophysiological ambulatory monitoring," European Journal of Psychological Assessment, vol. 23, no. 4, pp. 214-226, 2007.

[14] D. De Beurs et al., "The role of mobile phone technology in understanding and preventing suicidal behavior," 2015.

[15] T. Dehling et al., "Exploring the Far Side of Mobile Health: Information Security and Privacy of Mobile Health Apps on iOS and Android," JMIR mHealth and uHealth, vol. 3, no. 1, 2015.

[16] K. Huckvale et al., "Unaddressed Privacy Risks in Accredited Health and Wellness Apps: A Cross-Sectional Systematic Assessment," BMC medicine, vol. 13, no. 1, p. 214, 2015.

[17] B. Zapata et al., "Mobile PHRs Compliance with Android and iOS Usability Guidelines," Journal of Medical Systems, vol. 38, no. 8, p. 81, 2014.

[18] A. Azfar et al., "Forensic Taxonomy of Popular Android mHealth Apps," arXiv preprint arXiv:1505.02905, 2015.

[19] H. Seabrook et al., "Medical Applications: a Database and Characterization of Apps in Apple iOS and Android Platforms," BMC research notes, vol. 7, no. 1, p. 573, 2014.

[20] B. Zapata et al., "Empirical Studies on Usability of mHealth Apps: A Systematic Literature Review," Journal of medical systems, vol. 39, no. 2, p. 1, 2015.

[21] Z. Benenson et al., "Android and iOS Users' Differences Concerning Security and Privacy," in Extended Abstracts on Human Factors in Computing Systems. ACM, 2013, pp. 817-822.

[22] H. Ubhi et al., "A comparison of the characteristics of ios and android users of a smoking cessation app," Translational Behavioral Medicine, vol. 7, no. 2, pp. 166-171, 2017.

[23] F. Götz et al., "Users of the main smartphone operating systems (iOS, Android) differ only little in personality," PloS one, vol. 12, no. 5, pp. $1-18,2017$.

[24] M. Zappatore et al., "Mobile crowd sensing-based noise monitoring as a way to improve learning quality on acoustics," in 2015 International Conference on Interactive Mobile Communication Technologies and Learning (IMCL). IEEE, 2015, pp. 96-100.

[25] Z. Qin and Y. Zhu, "Noisesense: A crowd sensing system for urban noise mapping service," in 22nd International Conference on Parallel and Distributed Systems. IEEE, 2016, pp. 80-87.

[26] G. Galvez et al., "Feasibility of ecological momentary assessment of hearing difficulties encountered by hearing aid users," Ear and hearing, vol. 33, no. 4, p. 497, 2012.

[27] B. Martínez-Pérez, "Mobile health applications for the most prevalent conditions by the world health organization: review and analysis," Journal of medical Internet research, vol. 15, no. 6, 2013.

[28] T. Bright and D. Pallawela, "Validated smartphone-based apps for ear and hearing assessments: a review," JMIR Rehabilitation and Assistive Technologies, vol. 3, no. 2, 2016.

[29] H. Ma et al., "Opportunities in mobile crowd sensing," IEEE Communications Magazine, vol. 52, no. 8, pp. 29-35, 2014.

[30] K. Agrawal et al., "Towards incentive management mechanisms in the context of crowdsensing technologies based on trackyourtinnitus insights," Procedia computer science, vol. 134, pp. 145-152, 2018.

[31] R. Pryss et al., "Mobile Crowdsensing Services for Tinnitus Assessment and Patient Feedback," in IEEE Int'l Conf on AI \& Mobile Services. IEEE, 2017, pp. 22-29.

[32] M. Bradley and P. Lang, "Measuring Emotion: The Self-Assessment Manikin and the Semantic Differential," Journal of Behavior Therapy and Experimental Psychiatry, vol. 25, no. 1, pp. 49-59, 1994. 